\title{
The Truth behind the Solar System in the Universe
}

\author{
Rami Ayoob \\ University of Bahrain, Manama, Kingdom of Bahrain \\ Email: rayoob@hotmail.com
}

How to cite this paper: Ayoob, R. (2021) The Truth behind the Solar System in the Universe. Advances in Aerospace Science and Technology, 6, 158-169.

https://doi.org/10.4236/aast.2021.63011

Received: August 2, 2021

Accepted: September 3, 2021

Published: September 6, 2021

Copyright $\odot 2021$ by author(s) and Scientific Research Publishing Inc. This work is licensed under the Creative Commons Attribution-NonCommercial International License (CC BY-NC 4.0).

http://creativecommons.org/licenses/by-nc/4.0/

\begin{abstract}
This research focuses on multiple facts regarding the earth gravity and the space mechanism, mainly on the solar systems including the Sun and the planets belonging to it. Our solar system consists of our star, the Sun, and everything bound to it by gravity based on Albert Einstein and Isaac Newton theories. The planets are Mercury, Venus, Earth, Mars, Jupiter, Saturn, Uranus, Neptune and Pluto, dozens of moons, millions of asteroids, Comets and meteoroids [1]. Also, it will discuss about The Geocentric model and how scientifically proofed that the Earth is not orbiting the sun as it has a fixed position in the universe with the rotation around its axis and the sun is orbiting the Earth in one solar year. The output of the Geocentric model led to that the gravity is a feature generated by the planet itself to be measured reference to the weight granted to the matter.
\end{abstract}

\section{Keywords}

Astronomy, Deep Space, Earth, Sun, Galaxy, Gravitation, Heliocentric Model, Geocentric Model, Orbit, Planet, Solar System, Space Exploration, Sidereal

\section{Introduction}

Researches are always measured by logic and experiments, as a fact, humans build their results based on what they discovered and observed within their environment.

All the analyses are built on what we learned and what the human brains can understand it, and this knowledge is an output from the place where we live, as in the same environment the humans and other Creatures are learning from each other and create rules based on best practices and best results that they got from these practices. What we learn and practice in the Sea is differed than what 
we learn in the Desert, Jungle or on the Mountain, even countries are different from each other, and every environment has its own rules, creatures, plants, living style and culture.

Looking to the Earth Gravity, it's a tangible force provided to the objects on its surface and the other matters around it for the stability and continuity to their functions.

Any object on the earth has the same speed of the earth rotation speed at any layer or altitude, granted from this rotation (with consideration to the distance from the core). This object is sustained on the earth with Zero speed as long it's not producing another speed from walking for the human, engines for the cars and any other forces generated by the object itself or by other object.

The geocentric model, in which planet Earth is the center of the universe and is circled by the sun and all the planets, had been the accepted cosmological model since ancient times. By late antiquity, this model had come to be formalized by ancient Greek and Roman astronomers, such as Aristotle (384 - 322 $\mathrm{BCE}$ ), who's theories on physics became the basis for the motion of the planets, and Ptolemy, who proposed the mathematical solutions.

The geocentric model essentially came down to two common observations. First of all, to ancient astronomers, the stars, the sun, and the planets appeared to revolve around the Earth on daily basis. Second, from the perspective of the Earth-bound observer, the Earth did not appear to move, making it a fixed point in space.

In the 16th century, Nicolaus Copernicus began devising his version of the heliocentric model, which represented the culmination of year's worth of research. Like others before him, Copernicus built on the work of number classical astronomers who did not support the geocentric view [2].

And this belief (the Heliocentric Model) is continued with the next scientists up to the day. And this is what will be discussed in this research, why do we need to consider the Geocentric as a base model for our universe.

\subsection{Method}

1) The method used in this research is an analysis on the given data from multiple resources such as Space Agencies, Astronomy and physics authorities, mainly from their web sites.

2) Physics and mathematical formulas been involved to process and compare the given data as mentioned above, such as Newton's Law of Motion, $F=M^{\star}$ $A$.

3) Beside to the self study and monitoring of the earth sphere, supported with shots of the stars and planets.

\subsection{Purpose}

1) To provide a clear understanding of the Earth Gravitational and Geocentric Phenomenon, the causes and the relation with other influences and objects, such 
as the stars, planets and mainly the sun.

2) To discuss the existing theories and formulas those belongs to this subject and connect them with other facts and inputs.

3) To come out with new or amended Theory and Formula that might lead to significant changes on physics science and Space exploration.

\section{Gravitational Theory}

This Chapter will discuss and explain the recent Theories of the gravity law's in the Modern History, which established the current Physics and Astronomy Sciences, There are two main Roots in this field as shown below:

\subsection{Sir Isaac Newton (1643-1727)}

Newton came up with the theory of gravity instantly, when an apple fell from a tree and hit him on the head. And it got him to thinking about the mysterious force that pulls objects to the ground. Stating that the more mass an object had, the more it attracted other objects [3].

Newton's law of gravitation, statement that any particle of matter in the universe attracts any other with a force varying directly as the product of the masses and inversely as the square of the distance between them. $F=G\left(m_{1} m_{2}\right) / R^{2}$ [4] $G$ $=6.673 \times 10^{-11} \mathrm{~N} \cdot \mathrm{m}^{2} / \mathrm{kg}^{2}[5]$.

From this law, we can calculate the earth gravity as: $g=G^{\star} M_{\text {earth }} / R^{2}=9.8$ $\left(\mathrm{m} / \mathrm{s}^{2}\right)$ Earth Surface.

Newton's Law of Motion (second law), the acceleration of an object as produced by a net force is directly proportional to the magnitude of the net force, in the same direction as the net force, and inversely proportional to the mass of the object. $F=M^{*} A[6]=W($ weight $)=M^{*} 9.8\left(\mathrm{~m} / \mathrm{s}^{2}\right)$ Earth Surface.

\subsection{Albert Einstein (1879-1955)}

As a German-born theoretical physicist who developed the theory of relativity, one of the two pillars of modern physics.

Gravity is most accurately described by the general theory of relativity (proposed by Albert Einstein in 1915), which describes gravity not as a force, but as a consequence of the curvature of space time caused by the uneven distribution of mass [7].

As he worked out the equations for his general theory of relativity, Einstein realized that massive objects caused a distortion in space-time. Imagine setting a large body in the center of a trampoline. The body would press down into the fabric, causing it to dimple. A marble rolled around the edge would spiral inward toward the body, pulled in much the same way that the gravity of a planet pulls at rocks in space.

One of the most famous equations in mathematics comes from special relativity. The equation $E=m{ }^{*} c^{2}$ means "energy equals mass times the speed of light squared". 


\section{Research Conclusion}

In this chapter, I will explain the other aspect of the gravity phenomenon after gathering and connecting multiple factors together, which led to other theory, differs than the other two mentioned earlier.

\subsection{The Sidereal Day Statistics}

Heliocentric model was built on several theories as explained above, one of the key factor to make this model doable is the Sidereal day and solar day as shown in Figure 1 below:

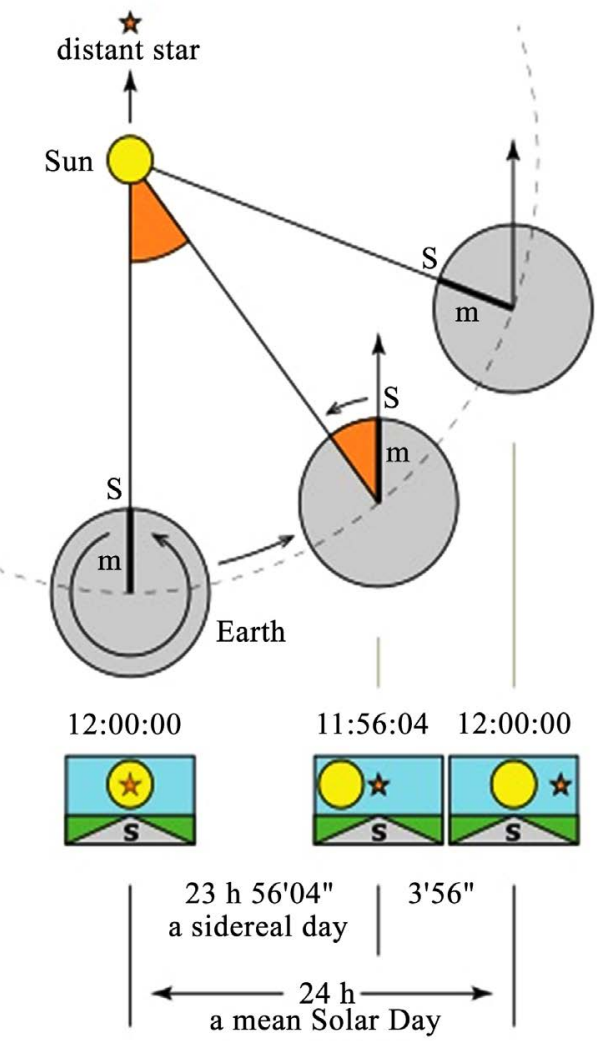

Figure 1. The sidereal day.

As shown in the figure that the sidereal day against to the distant stars is $23 \mathrm{~h}$ $56 \mathrm{~m}$ and the solar day is $24 \mathrm{~h}$ [8]. So to maintain the solar day $24 \mathrm{~h}$, Earth makes one rotation around its axis in a sidereal day. During that time it moves a short distance (about $1^{\circ}$ ) along its orbit around the Sun. So after a sidereal day has passed, Earth still needs to rotate slightly more before the Sun reaches local noon according to solar time. A mean solar day is, therefore, nearly 4 minutes longer than a sidereal day [9].

This statistics is not accurate according to several facts as shown below:

1) Let's assume the earth is rotating in straight track in Figure 2, as an example to simulate the earth orbit at different track models for rotation behavior clarification. 
Day $3=23: 54 \mathrm{H}$

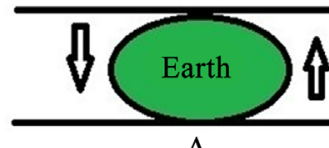

One Complete Rotation
Day $2=23: 54 \mathrm{H}$

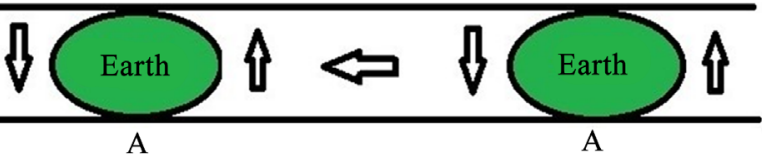

One Complete Rotation
One Complete Rotation

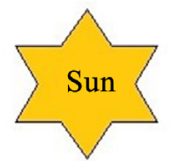

Figure 2. Straight track orbit.

If we bend the orbit in Figure 3.

Day $2=23: 54 \mathrm{H}$

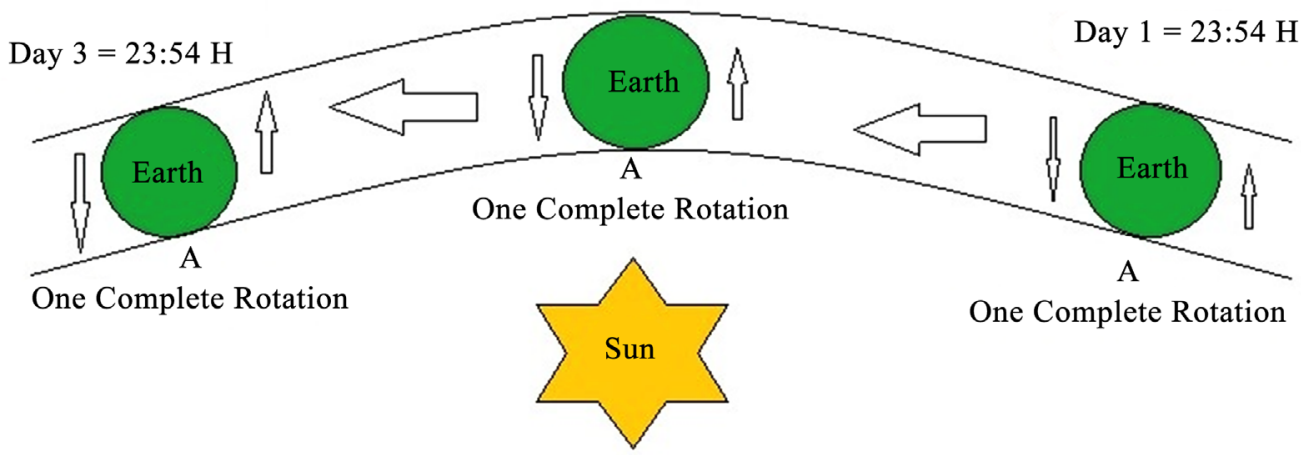

Figure 3. Bended track orbit.

The earth also will complete one rotation around its Axis from A-A in 23:54 H As a result, the earth will not moves a short distance (about $1^{\circ}$ ) every day to maintain the solar day $24 \mathrm{~h}$.

2) Another fact, if we look to the moon as its orbits the earth and rotate around its Axis once every Month [10]. And let's do the same assumptions we did with the earth above in Figure 4.

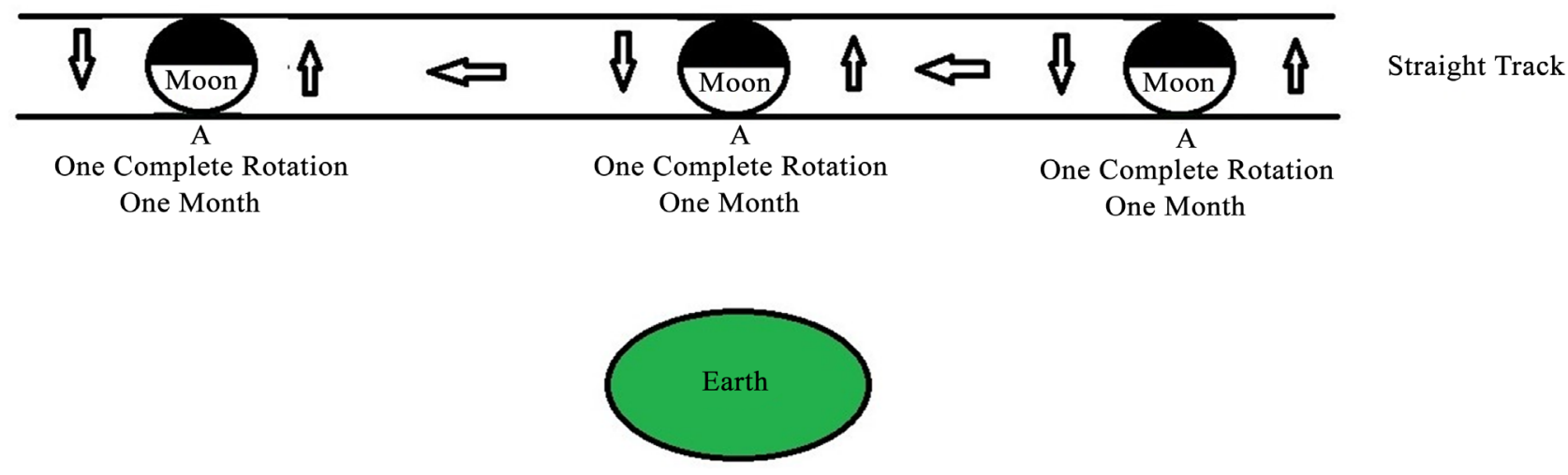

Figure 4. Straight track orbit. 
The Moon will complete one rotation around its Axis from A-A in one month in a straight track.

And if we bend the orbit in Figure 5.

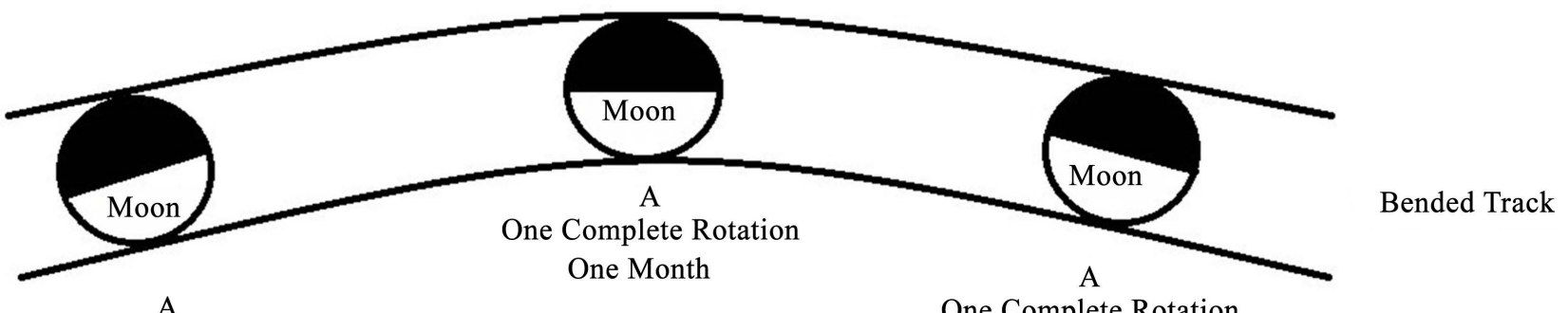

One Complete Rotation

One Month

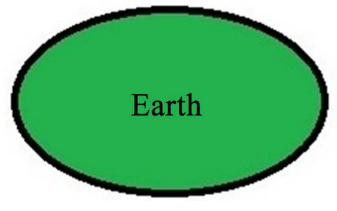

Figure 5. Bended track orbit.

The Moon will also complete one rotation around its Axis from A-A in one month.

And this is why we get the same face side of the moon around the month while it's orbiting the Earth as show in Figure 6.

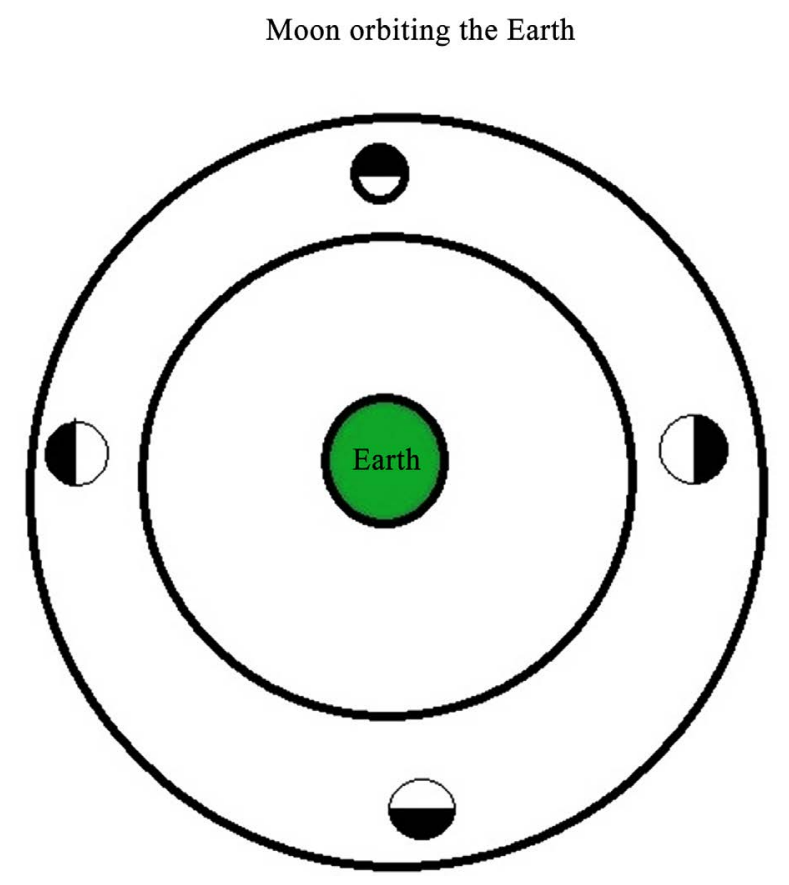

Figure 6. Moon orbit the earth.

This is the behavior of the planets including the earth while they are rotating around their axis. 
So Sidereal day theory with earth movement by less one degree to maintain the Solar day is not correct in Figure 7.

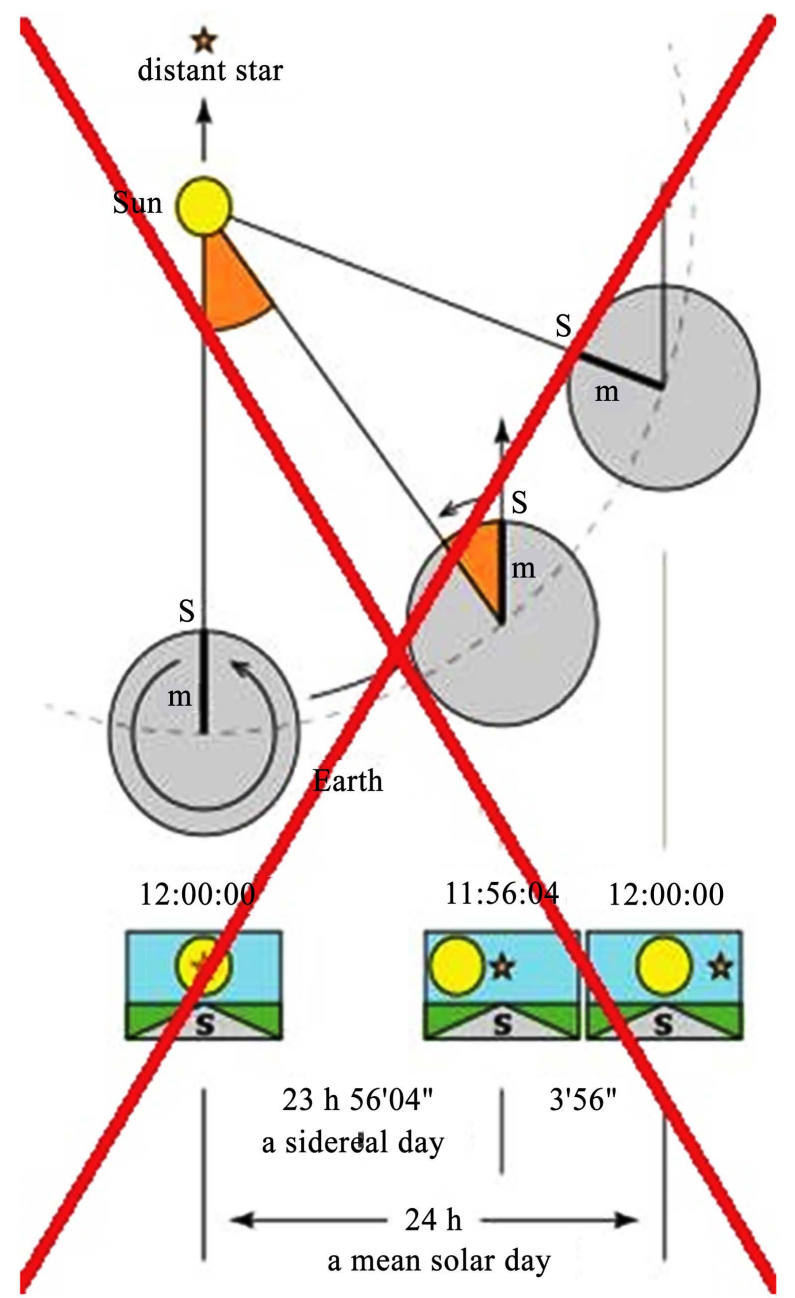

Figure 7. Sidereal day.

\subsection{The Geocentric Model vs. the Heliocentric Model}

Based on the current statistics, the earth rotate around its axis once every 23.9 Hours at speed of $1676.56 \mathrm{~km} / \mathrm{h}$, and the circumference of the earth is $40,074.16$ $\mathrm{km}$ [11].

The Earth orbit the sun in 365.25 Days at speed of $107,280 \mathrm{~km} / \mathrm{h}$, and the circumference of this orbit is 940 Millions/km [12].

Linking the above statistics, we realize that the earth is finishing a complete one rotation in 23 hours and $56 \mathrm{~min}$, by -4 minutes every day of the length of the day which is 24 Hours. For example, if today the sun rise at 6 am at certain location on the earth, tomorrow the sun will rise at $6 \mathrm{am}-4$ minutes at the same location, same scenario every day by $-4 \mathrm{~min}$.

Let's apply the explained fact in (3.1) to the heliocentric model as below in Figure 8. 
Day $3=23: 56 \mathrm{H}$

Day $2=23: 56 \mathrm{H}$

Day $1=23: 56 \mathrm{H}$

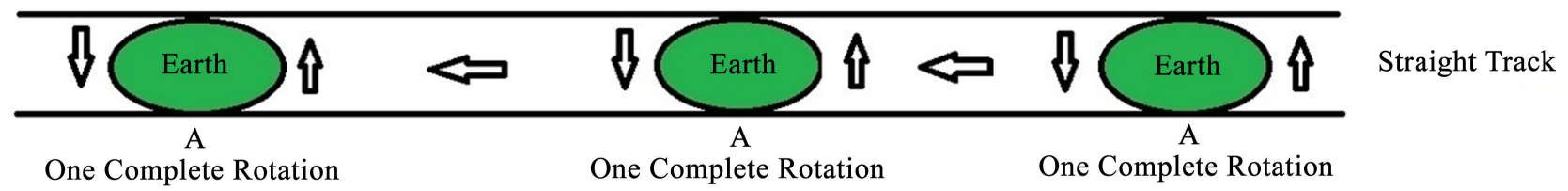

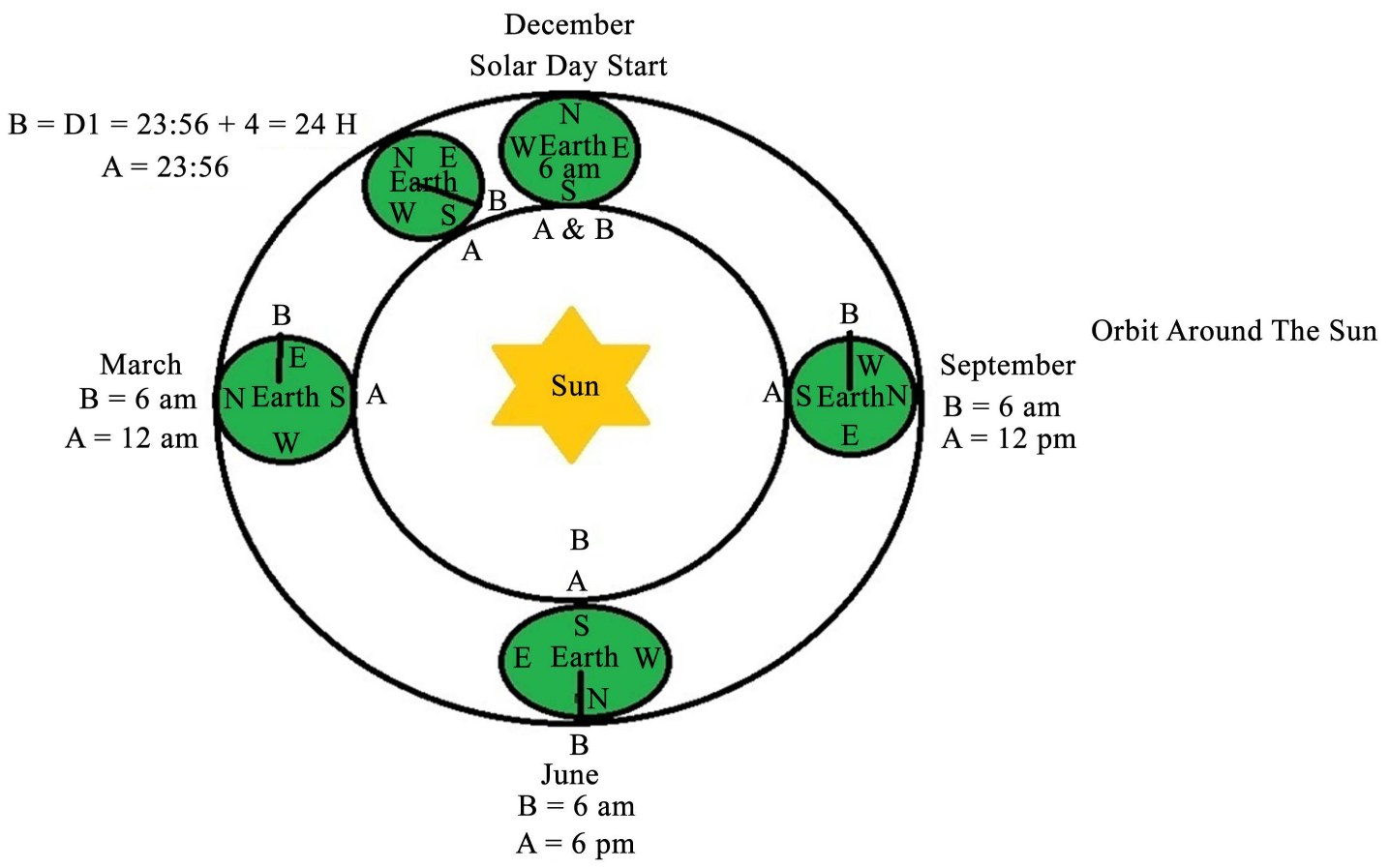

Figure 8. The heliocentric model.

If we consider the current solar system methodology that the earth orbiting the sun, we will end up with that at same location of the above example, the sun will rise some time at 12 am and some time will set at $12 \mathrm{pm}$, or some time will rise at $4 \mathrm{pm}$ and will set at $4 \mathrm{am}$, Likewise onwards all the year, and this is never happened at the history of the human been or at the time of the earth creation.

So the Heliocentric model is not applicable with the above data, as the earth speed not matching the day length (Solar Day), which is aligned with the sun rising and set, and led to 24 Hours duration as per to the time invention [13].

Geocentric is the only model can match the above data and keep the balance for the day time ( 24 Hours), with + or -2 to 3 Hours between the winter and summer in the average of the earth locations (Equatorial line for example). By applying the above data, the Sun will orbit the earth at the same circumference of 940 Millions $/ \mathrm{km}$ at the same speed of $107,280 \mathrm{~km} / \mathrm{h}$ which was considered for the earth [12].

The earth rotate around its axis once every 23.9 Hours at speed of $1041 \mathrm{mph}$ $(1676.56 \mathrm{~km} / \mathrm{h})$, and the circumference of the earth is $40,074.16 \mathrm{~km} \mathrm{[12].}$

To maintain the -4 minutes different of the rotation speed with the day length (24 Hours) the sun will move over its orbit: $((107,280 \mathrm{~km} / \mathrm{h}) \times 23.9 \mathrm{~h})=$ $2,563,992 \mathrm{~km}$. 
So the earth rotation speed must be aligned with the sun orbit speed per day, and by applying the below equation:

Earth circumference $\times$ Sun orbit speed $=$ Sun Daily movement distance $\times$ Earth Rotation Speed.

Applying the data to the equation:

Earth Rotation Speed $=1676.74 \mathrm{~km} / \mathrm{h}$.

And this is the recent record of the earth rotation speed.

Figure 9 will explain the applied data.

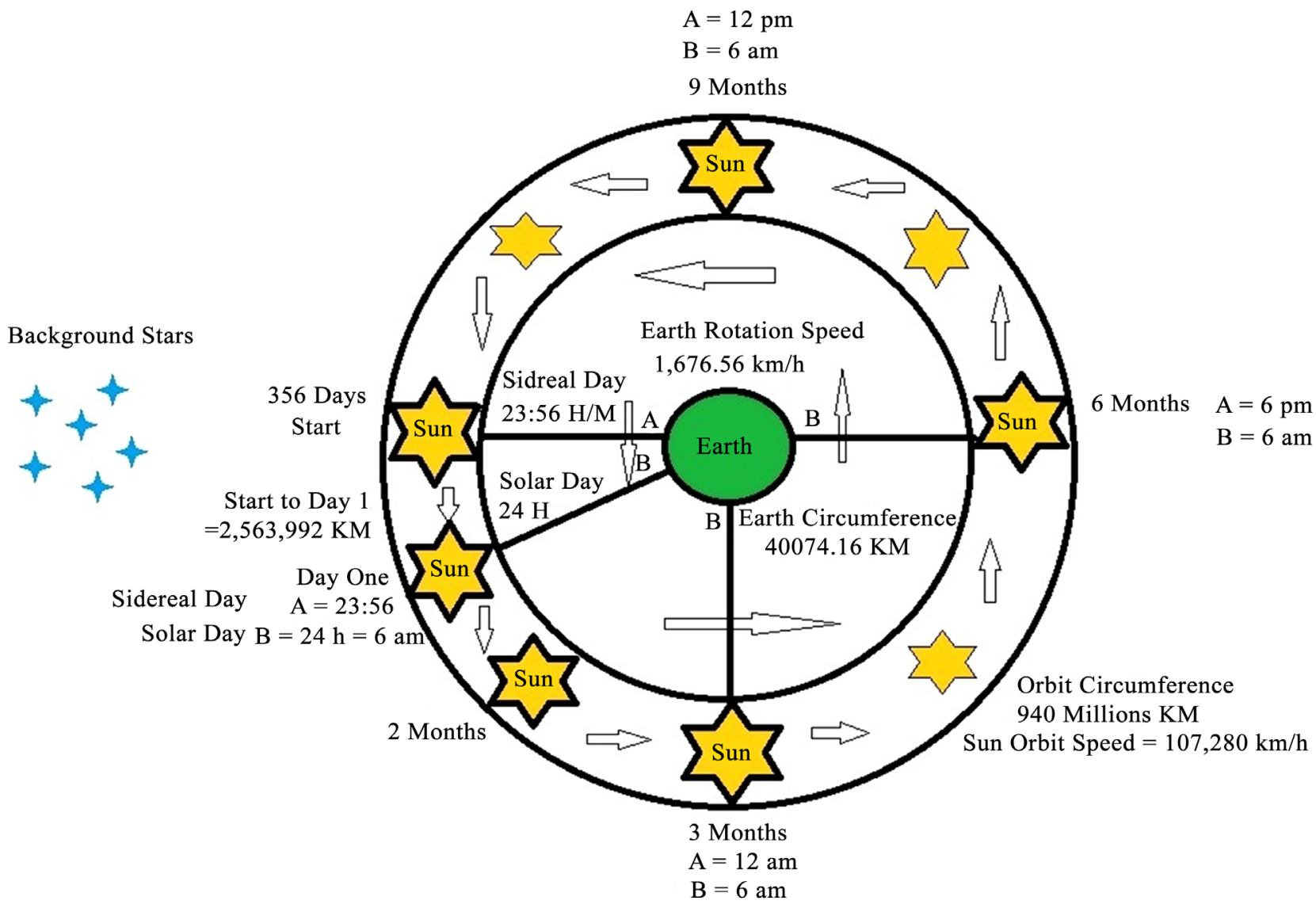

Figure 9. The geocentric model.

\subsection{A Potential Energy Generated by the Earth from the Rotation around Its Axis}

Based on the above Conclusion $3.1 \& 3.2$, the gravity can be assessed as below:

This Energy generated by the Earth from the rotation around its axis is granted to all objects on the earth including humans and other masses, it grab the objects to the earth surface, as well to the other matters in the atmosphere (7 layers) and the earth (5 layers).

This energy is generated by the rotation speed regardless to the other factors such as the mass, density or diameter of the earth, as this rotation is the direct impact on the object itself.

With the observation to this rotation we come out that the earth form elliptic- 
al shape with (Equatorial diameter: 12,756 km) and (Polar diameter: 12,714 km) with almost $42 \mathrm{~km}$ different. As this shape is formed from this rotation were the energy generated bend the earth on the poles and stretch it on the equator (these details can be simulated by any rotation experiment: e.g., rubber ball).

As an example, when we drive the car at high acceleration, our body will resist the force of this acceleration until the car reduce its acceleration or move at constant speed, then the objects inside the car will have the same speed of the car itself with no resistance to the car speed, and we can move freely within the car itself as long it moves in the same direction at the same speed with no other external forces (rough roads, winds, or any). Same thing for the train and airplane, when the plane at fixed altitude, speed and direction, we can move freely to anywhere in the plane as we are on the ground.

Since the earth has constant speed and there are no tangible forces of other object from the outer space effecting directly on the earth objects, so far any masses on the earth can move to any four direction (E.W.N.S) freely with consideration to the environmental resistance such as wind, wave, surface type and any other factors, which mean the object will not get sudden impact or forces generated from the earth as this force is stored in the object itself.

Based on the above, the earth grabs the objects with direction to its core and grant speed to them by the force from its rotation, as the core represent the center of the earth rotation, the force is gradually reduced by the distance of the object away from the earth core.

If this rotation is not exists, we will not granted any forces to our bodies. So far the correct formula of the gravity shall be obtained as below:

$$
g_{2}=g_{1} * W_{2} / W_{1}
$$

where $g_{1}=9.8\left(\mathrm{~m} / \mathrm{s}^{2}\right)$ earth gravity, $W_{1}=$ the object weight on the earth surface without any external forces, $W_{2}=$ the object weight at other planet or at other environmental effects (wind, wave, altitude or any), $g_{2}=$ the gravity at other planet or at other environmental effects (wind, wave, altitude or any)

\subsection{As a Result}

By connecting all the inputs above there are TWO Facts related to the gravity and solar system:

1) Based on Conclusion $3.1 \& 3.2$, the Earth is not orbiting the sun as it has a fixed position in the universe with the rotation around its axis. Due to the events accruing on the earth such as the four seasons, the Sun and the Moon are orbiting the earth at the same duration, degree and direction as provided by the recent science. So far with these facts, the gravitational theory of a consequence of the curvature of space time caused by the uneven distribution of mass and the theory that any particle of matter in the universe attracts any other with a force varying directly as the product of the masses are not valid. The planets including the earth will generate their own gravity from the rotation around their axis with the consideration to the other physics factors on other planets, as this fact only 
applicable on the earth and just a theory for the other planets as well for the records collected earlier by Astronomer's and space agencies.

2) The Earth generates direct Energy from the rotation around its axis to its objects in form of gravity, which gives these objects the weights to sustain on the earth with the direction to its core. This force shall be measured by weight not by Mass, as the mass is fixed and the weight is variable depend on the gravity.

The unit measure shall be standard at the earth surface and take it as a reference for other measures in different locations using the below formula:

$$
g_{2}=g_{1} * W_{2} / W_{1}
$$

where $g_{1}=9.8\left(\mathrm{~m} / \mathrm{s}^{2}\right)$ earth gravity, $W_{1}=$ the object weight on the earth surface without any external forces, $W_{2}=$ the object weight at other planet or at other environmental effects (wind, wave, altitude or any), $g_{2}=$ the gravity at other planet or at other environmental effects (wind, wave, altitude or any).

\section{Summary}

As a result, Space is so wide and every planet, star or any other object has its own rules, physics, chemistry and other sciences, as we cannot apply what we learned and practiced on the earth to another planet, star or space unless if the humans can live there and do the same learning path as what they did on the earth and come out with different sciences than what they produced on the earth. But human's capabilities only allow him to learn from the place they born and the events around him.

Gravity is what we feel it and what gives us the weight to live and move on the earth, so only the earth can produce that force to be stored in our bodies and make the balance of live on its surface and other atmosphere and inner layers. We can only apply the rules and physics what we discovered, tested and simulated, and that what I explained it on the above about the reality of the Earth Gravity in Conclusion 3.3. Then, we can practice on the other planets and compare the results if there are any matches with our results by practice not by assumptions.

As well for the SUN, it's orbiting the earth to provide us the four seasons and other events, as it's fit for that purpose. The sidereal Day and Solar Day in the Heliocentric model will not be fit, as the earth will complete one full rotation around its axis every day to the same point facing the sun as explained in Conclusion $3.1 \& 3.2$.

Finally, humans must be concentrated on the environment where do they live and the events happening around them, build the results by logic and by what we do practice, connect the outputs together and get out with tangible facts where human being can understand and learn from them.

\section{Conflicts of Interest}

The author declares no conflicts of interest regarding the publication of this paper. 


\section{References}

[1] Glaze, L. (2021) Our Solar System.

https://solarsystem.nasa.gov/solar-system/our-solar-system/overview/

[2] Williams, M. (2016, January 11) What Is the Geocentric Model of the Universe? https://www.universetoday.com/32607/geocentric-model/

[3] Spirko, J. (2017, April 25) The Discovery of Gravity \& the People Who Discovered It. https://sciencing.com/discovery-gravity-people-discovered-16994.html

[4] The Editors of Encyclopaedia Britannica (2021) Newton's Law of Gravitation. https://www.britannica.com/science/Newtons-law-of-gravitation

[5] Physical Classroom (n.d.) Newton's Law of Universal Gravitation. https://www.physicsclassroom.com/class/circles/Lesson-3/Newton-s-Law-of-Univer sal-Gravitation

[6] Sands, K. (2021, August 10) Newton's Laws of Motion. https://www1.grc.nasa.gov/beginners-guide-to-aeronautics/newtons-laws-of-motio $\underline{\mathrm{n} /}$

[7] Wikipedia (n.d.) Gravity. https://en.wikipedia.org/wiki/Gravity

[8] Weisstein, E.W. (1996-2007) Sidereal Day. https://scienceworld.wolfram.com/astronomy/SiderealDay.html

[9] Wikipedia (n.d.) Sidereal Time. https://en.wikipedia.org/wiki/Sidereal time

[10] Taylor Redd, N. (2021, June 17) Does the Moon Rotate? https://www.space.com/24871-does-the-moon-rotate.html

[11] Howell, E. (2018, June 23) How Fast Is Earth Moving? https://www.space.com/33527-how-fast-is-earth-moving.html

[12] Williams, D.R. (2019, October 21) Planetary Fact Sheet. https://nssdc.gsfc.nasa.gov/planetary/factsheet/

[13] Basu, J. (2018, October 30) Why There Are 24 Hours in a Day and 60 Minutes in an Hour.

https://medium.com/@jothibasuofficial/why-there-are-24-hours-in-a-day-and-60minutes-in-an-hour-b670879cbe99 